\title{
$\alpha-\mathrm{MoO}_{3}$ 纳米带的制备及模拟燃油光催化氧化脱硫活性
}

\author{
甄延忠 ${ }^{1,2}$, 李 静 $^{2}$, 王丹军 ${ }^{2}$, 付 峰 $^{2}$, 薛岗林 ${ }^{1}$
}

(1. 西北大学 合成与天然功能分子化学教育部重点实验室, 西安 $710069 ; 2$. 陕西省化学反应工程重点实验室, 延 安 716000)

摘 要: 采用简单的水热法获得了一维结构的 $\alpha-\mathrm{MoO}_{3}$ 纳米带, 利用 XRD、SEM、TEM、EDS 和 UV-Vis-DRS 光谱 等测试手段对其相组成、形貌、纯度和光吸收特性进行表征。以噻吩模拟含硫污染物探讨了 $\alpha-\mathrm{MoO}_{3}$ 纳米带对模拟 FCC 汽油的光催化氧化脱硫( Photo-Cat-ODS) 活性及其光催化氧化脱硫机理。结果表明: 一维 $\alpha-\mathrm{MoO}_{3}$ 纳米带有利 于抑制光生电子和空穴的复合, 对模拟 FCC 汽油光催化脱硫活性远高于商品 $\mathrm{MoO}_{3}$ 和商品二氧化钛(P25), 可见光 光照 $240 \mathrm{~min}$ 模拟汽油 FCC 的脱硫率达到 $86.9 \%$ 。

关 键 词: 水热法; $\alpha-\mathrm{MoO}_{3}$ 纳米带; 光催化氧化; 脱硫

中图分类号: TQ174 文献标识码: A

\section{Synthesis of $\alpha-\mathrm{MoO}_{3}$ Nanobelt and Its Photocatalytic Oxidative Desulfuriza- tion(Photo-ODS) Activity of Simulation Fuel}

\author{
ZHEN Yan-Zhong $^{1,2}$, LI Jing ${ }^{2}$, WANG Dan-Jun ${ }^{2}$, FU Feng ${ }^{2}$, XUE Gang-Lin ${ }^{1}$ \\ (1. Key Laboratory of Synthetic and Natural Functional Molecule Chemistry of the Ministry of Education, Northwest Univer- \\ sity, Xi'an 710069, China; 2. Shaanxi Key Laboratory of Chemical Reaction Engineering, Yan'an 716000, China)
}

\begin{abstract}
D} \alpha-\mathrm{MoO}_{3}$ nanobels were prepared by facile hydrothermal synthesis. Phase, morphology, purity and optical adsorption properties of as-synthesized samples were investigated by XRD, SEM, TEM, EDS and UV-Vis-DRS spectra. Furthermore, thiophene was selected as a typical pollutant of simulated fuel to evaluate the photocatalytic oxidative desulfurization (Photo-Cat-ODS) activity of as-prepared $1 \mathrm{D} \alpha-\mathrm{MoO}_{3}$ nanobelt and the mechanism for the photocatalytic oxidation of thiophene was explored. The photocatalysis experimental results indicate that photocatalytic oxidative desulfurization activity of $1 \mathrm{D} \alpha-\mathrm{MoO}_{3}$ nanobelts is better than that of purchased $\mathrm{MoO}_{3}$ and $\mathrm{P} 25$ due to their reducing recombination of photogenerated electron-hole pairs. The desulfurization rate of simulated FCC fuel reaches $86.9 \%$ under visible light irradiation for $240 \mathrm{~min}$.
\end{abstract}

Key words: hydrothermal synthesis; $\alpha-\mathrm{MoO}_{3}$ nanobelt; photocatalytic oxidative; desulfurization

近年来，随着汽车工业的快速发展，汽车尾气 排放的 $\mathrm{SO}_{x}$ 造成了严重的环境污染 ${ }^{[1-2]}$, 因此降低油 品中硫化合物的含量受到了国内外研究人员的广泛
关注。在众多的脱硫工艺中, 催化氧化脱硫(ODS) 因反应条件温和、投资少, 不消耗 $\mathrm{H}_{2}$, 且对加氢脱 硫(HDS)难以脱除的二苯并噻吩系列有很好的脱除

收稿日期：2014-10-14; 收到修改稿日期：2014-12-18

基金项目：陕西省科技统筹创新工程(2012KTCL03-16); 陕西省教育厅专项项目(12JS117，14JK1831); 陕西省高水平大学建设专 项资金(2013SXTS03)

Innovation Project of Science and Technology of Shaanxi (2012KTCL03-16); Special Projects of Shanxi Provincial Education Office (12JS117, 14JK1831); Special Fund Projects High-level University Constraction of Shanxi (2013SXTS03)

作者简介: 甄延忠(1979-)，男，博士研究生. E-mail: zyz943@163.com

通讯作者: 薛岗林，教授. E-mail: glxue@nwu.edu.cn 
效果，而被誉为绿色的脱硫工艺。近年来兴起的光 催化氧化脱硫 (Photo-Cat-ODS) 技术, 作为一种新 型的催化氧化脱硫技术倍受人们关注 ${ }^{[3-10]}$ 。

$\mathrm{MoO}_{3}$ 作为一种 $\mathrm{n}$ 型宽带隙半导体材料, 以其独 特的结构和稳定的性能, 广泛应用于电极材料、催 化、光致变色、电致变色及其传感器等领域 ${ }^{[11-15]}$ 。 在光催化领域, $\mathrm{MoO}_{3}$ 可同时响应紫外和可见光的光 学特性, 引起广泛关注 ${ }^{[16]}$ 。Shao 等 ${ }^{[17]}$ 制备了单晶结 构的一维(1D) $\alpha-\mathrm{MoO}_{3}$ 纳米棒, 在可见光照射 $1 \mathrm{~h}$ 后, 该材料对罗丹明 B (RhB) 降解率为 $100 \%$ 。Kang 等 ${ }^{[16]}$ 利用 $1 \mathrm{D} \mathrm{MoO}_{3}$ 纳米棒在紫外-可见光区成功地光催 化降解甲苯胺蓝和氯苯。 $\mathrm{Hou}$ 等 ${ }^{[18]}$ 利用 $\alpha-\mathrm{MoO}_{3}$ 纳 米带, 在可见光区光催化降解亚甲基蓝(MB)。但有 关 $\alpha-\mathrm{MoO}_{3}$ 用于光催化氧化脱硫的研究还未见报道。

本工作采用水热法制备了单晶结构的 $\alpha-\mathrm{MoO}_{3}$ 纳米带, 通过 XRD、SEM、TEM、EDS 和 UVVis-DRS 等方法对其进行表征，并以噻吩的正辛烷 溶液模拟催化裂化 (FCC)汽油, 研究了 $\alpha-\mathrm{MoO}_{3}$ 纳 米带光催化氧化脱硫活性。

\section{1 实验方法}

\section{1 主要仪器和试剂}

全自动 X 射线粉末衍射仪(XRD-7000 型, 日本 岛津公司); 紫外分光光度计( UV-2550 型, 日本岛 津公司); TM3000 扫描电子显微镜(日本日立); JEM-2100 透射电镜(日本); 光化学反应仪(XPA-2 型, 南京胥江机电厂) ; 医用高速离心机(LG102.4A 型, 北京); 电子天平( FA1004N 型, 上海精密 科学仪器有限公司); 真空干燥箱( DZE-3 型, 上海 福玛设备有限公司)。

$\left(\mathrm{NH}_{4}\right)_{6} \mathrm{Mo}_{7} \mathrm{O}_{24} \cdot 4 \mathrm{H}_{2} \mathrm{O}$ (分析纯); $\mathrm{HNO}_{3}$ (含量 $61 \%$ 63\%, 分析纯); 噻吩 (分析纯); 正辛烷(分析 纯); 无水乙醇 $\left(\mathrm{CHCH}_{2} \mathrm{OH}\right.$, 分析纯); 商品 $\mathrm{MoO}_{3}$ (分 析纯); 商品二氧化钛 (P25); 实验用水为蒸馏水。

\section{2 一维 $\alpha-\mathrm{MoO}_{3}$ 纳米带的制备}

称取一定量的 $\left(\mathrm{NH}_{4}\right)_{6} \mathrm{MO}_{7} \mathrm{O}_{24} \cdot 4 \mathrm{H}_{2} \mathrm{O}$ 溶于 $10 \mathrm{~mL}$ 蒸馏水, 磁力搅拌, 待溶解完全后, 加入 $10 \mathrm{~mL} 2 \mathrm{~mol} / \mathrm{L}$ $\mathrm{HNO}_{3}$, 摚拌使其混合均匀, 将溶液转移至水热反应 罐中, 密封后放入烘箱, 升温至 $180^{\circ} \mathrm{C}$, 保持 $8 \mathrm{~h}$, 自 然冷却至室温, 将生成的浅蓝色沉淀离心沉降, 水 洗、醇洗，所得粉末置于真空干燥箱 $80^{\circ} \mathrm{C}$ 干燥 $6 \mathrm{~h}$ 。

\section{3 催化剂的表征}

采用日本岛津公司 XRD-7000 型全自动 X射线 粉末衍射仪 $(X R D)$ 鉴定样品的物相结构, $\mathrm{Cu} \mathrm{K \alpha}(\mathrm{Ni}$
滤玻片滤波, $\lambda=0.15418 \mathrm{~nm}$ ), 管电压 $40 \mathrm{kV}$, 管电 流 $30 \mathrm{~mA}$, 步长 $0.02^{\circ}$, 扫描范围 $2 \theta: 10^{\circ} \sim 80^{\circ}$, 扫描 速度 $1 \% \mathrm{~min}$ 。利用日本日立 TM3000 电子显微镜和 日本电子 JEM-2100 型透射电镜观察样品微观形貌。 采用日本岛津公司 UV-2550 型紫外可见分光光 度计测定粉体 UV-Vis 吸收光谱, 扫描范围 200 $800 \mathrm{~nm}$, 标准 $\mathrm{BaSO}_{4}$ 参比。

\section{4 氧化脱硫实验}

取 $0.5 \mathrm{~mL}$ 噻吩加入 $500 \mathrm{~mL}$ 正辛烷溶液中充分混 匀配制成模拟 FCC 汽油，其硫含量约为 $500 \times 10^{-6}$ 。 量取 $20 \mathrm{~mL}$ 上述溶液并称取一定量的催化剂加入到 $50 \mathrm{~mL}$ 石英试管中。为了检测生成产物, 在另一只 试管中再加入 $5 \mathrm{~mL} \mathrm{Ba}(\mathrm{OH})_{2}$ 稀溶液, 试管下部为水相, 上部为油相。将石英试管在黑暗中磁力搅拌 $30 \mathrm{~min}$, 达到吸附-脱附平衡，然后打开金卤灯照射，同时打 开旋转开关，保证受光均匀。每隔 $30 \mathrm{~min}$ 取样，然 后用乙腈进行萃取, 取其上清液在微库伦分析仪中 分析其硫含量的大小。在 WK-2D 型库仑分析仪上 进行含硫量的测定, 汽化室温度为 $680^{\circ} \mathrm{C}$, 燃烧室 温度为 $850^{\circ} \mathrm{C}$, 以氮气作为载气, 氧气为燃烧气, 碘 电极为参比电极, 铂电极为测量电极。按下式计算 脱硫率:

$$
\eta=\frac{C_{0}-C_{1}}{C_{0}} \times 100 \%
$$

$\eta$ 为脱硫率, $C_{0}$ 为原液中硫含量; $C_{1}$ 为反应后溶液 中硫含量。

\section{2 结果与讨论}

\section{1 催化剂的结构、组成和形貌分析}

图 1 是样品的 XRD 图谱, 由图可知, 各衍射峰

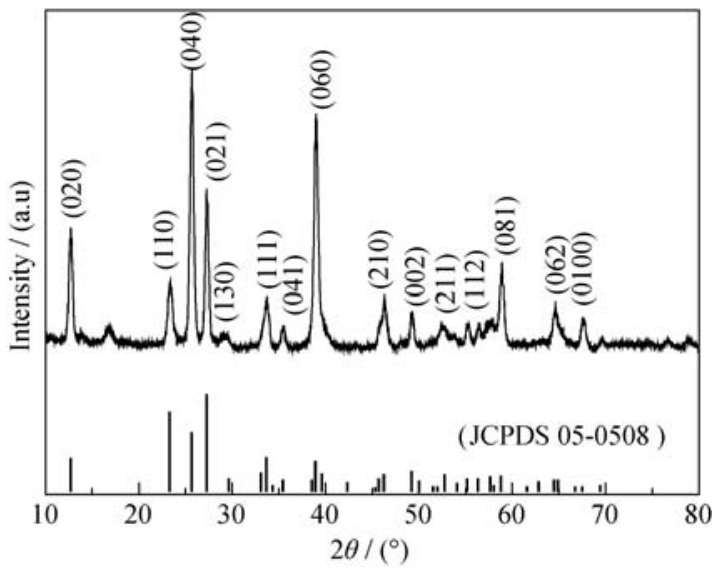

图 $1 \alpha-\mathrm{MoO}_{3}$ 纳米带的 XRD 图谱

Fig. 1 XRD patterns of as-synthesized $\alpha-\mathrm{MoO}_{3}$ nanobelts 
的位置和 $d$ 值与标准卡(JCPD 05-0508)一致, 可确 认样品为正交晶系的 $\alpha-\mathrm{MoO}_{3}$ 。XRD 图谱中没有出 现杂峰, 且衍射峰的强度较高, 说明样品纯度、结 晶度较高。与标准卡对比, 发现 $(02 k 0)$ 的衍射峰强 度明显增强, 说明样品生长具有各向异性 ${ }^{[17-18]}$ 。

用 Jade 6 软件进行结构精修, 计算得到样品的晶 胞参数为: $a=0.3957 \mathrm{~nm}, b=1.3839 \mathrm{~nm}, c=0.3688 \mathrm{~nm}$, $V=0.20193 \mathrm{~nm}^{3}$, 略小于标准卡 (JCPD 05-0508)中 的晶胞参数 $(a=0.3962 \mathrm{~nm}, b=1.3858 \mathrm{~nm}, c=0.3697 \mathrm{~nm}$, $\left.V=0.20299 \mathrm{~nm}^{3}\right)$ 。

图 2 是样品的 SEM 照片, 由此可见, 样品呈 一维带状结构。图 2(a)中插图为样品的 EDS 图谱, 由图谱可以看出样品由 Mo、O 两种元素组成, 原 子个数比 1:3, 不含其它杂质元素, 进一步表明所 得样品的纯度较高。图 3 是样品的 TEM 图谱, 由图 可知, 样品中 $\alpha-\mathrm{MoO}_{3}$ 纳米带的结构均匀, 单根纳米 带表面光滑, 带长约为 $10 \mu \mathrm{m}$, 带宽约 $0.2 \mu \mathrm{m}$ 。图 3 插图为对应纳米带的选区电子衍射图案(SAED), 其 衍射斑点为规则点阵, 说明样品为单晶结构, 在 SAED 中可以看出, 其中[001]方向垂直于纳米带的轴 向[010], 说明 $\alpha-\mathrm{MoO}_{3}$ 纳米带沿着 $[001]$ 方向生长 ${ }^{[18]}$ 。

\section{$2.2 \mathrm{UV}-\mathrm{Vis}-\mathrm{DRS}$ 分析}

图 3(a)为 $\alpha-\mathrm{MoO}_{3}$ 纳米带和商品 $\mathrm{MoO}_{3}$ (晶相为正 交晶系)的紫外-可见漫反射光谱(UV-Vis-DRS), 由 图可知, $\alpha-\mathrm{MoO}_{3}$ 纳米带和商品 $\mathrm{MoO}_{3}$ 均在紫外和 可见光区有明显的吸收, 其中 $\alpha-\mathrm{MoO}_{3}$ 纳米带的 吸收边界为 $420 \mathrm{~nm}$, 而商品 $\mathrm{MoO}_{3}$ 吸收边大约在 $450 \mathrm{~nm}$ 。 $\alpha-\mathrm{MoO}_{3}$ 纳米带的吸收边发生了明显的蓝 移, 归处于量子尺寸效应和表面效应。与微米级 $\mathrm{MoO}_{3}$ 相比较, $\alpha-\mathrm{MoO}_{3}$ 纳米带尺寸下降, 带隙变宽, 导致光吸收带向短波方向偏移 ${ }^{[19]}$; 此外, $\alpha-\mathrm{MoO}_{3}$ 纳 米具有较大的比表面积, 大的表面张力容易引起晶 格畸变, 导致晶格变小, 使其本征振动频率增大, 导 致光吸收带向短波方向偏移, 所以出现蓝移现象 ${ }^{[20]}$, 这与 XRD 分析结果一致。

根据半导体的带隙与带边吸收的关系: $\alpha \mathrm{h} v=$ $\left(\mathrm{h} v-E_{\mathrm{g}}\right)^{n[17,21]}$, 其中, $\alpha 、 \mathrm{~h} 、 v$ 和 $E_{\mathrm{g}}$ 分别是样品的吸 收系数、普朗克常数、光子频率和带隙, $\mathrm{MoO}_{3}$ 的 $n$ 值为 $1 / 2$, 估算样品的带隙值, 作 $(\alpha \mathrm{h} v)^{2}-\mathrm{h} v$ 图可得 $\alpha-\mathrm{MoO}_{3}$ 纳米带和商品 $\mathrm{MoO}_{3}$ 的带隙值分别为 3.2 和 $2.9 \mathrm{eV}$ (如图 3(b)所示)。
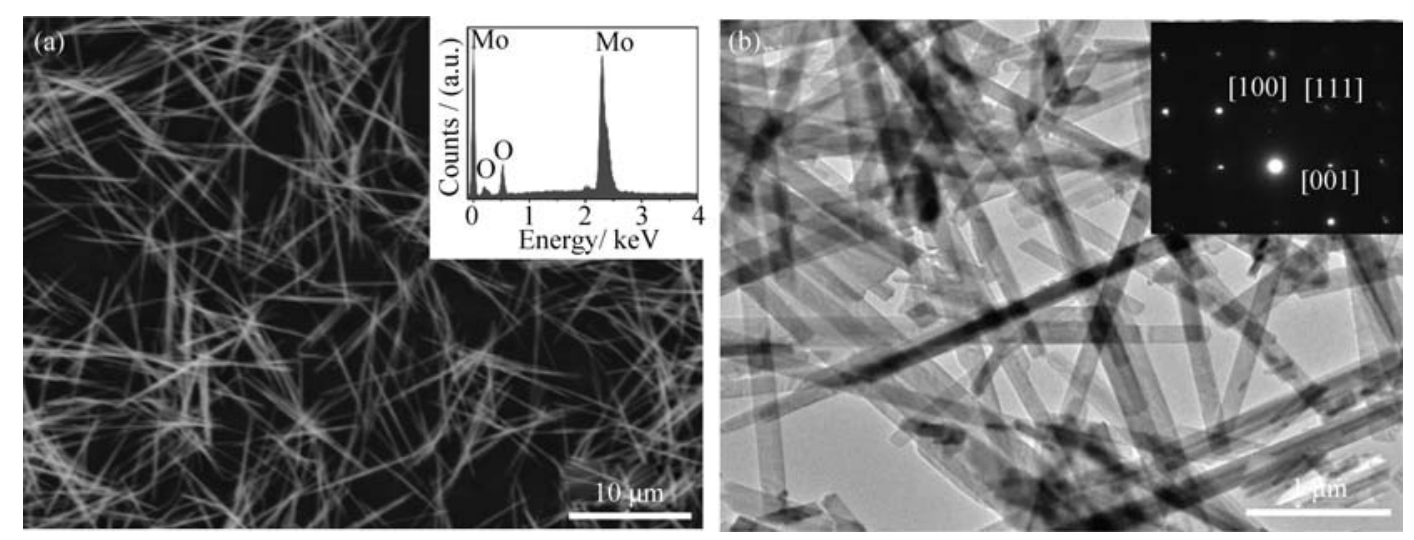

图 $2 \alpha-\mathrm{MoO}_{3}$ 纳米带的 SEM 照片(插图为 EDS 图谱)(a)和 TEM 照片(插图为 SAED 图谱)(b)

Fig. 2 SEM image (a) (with an inset showing the corresponding EDS spectrum) and TEM image (b) (with an inset showing the corresponding SAED pattern) of $\alpha-\mathrm{MoO}_{3}$ nanobelts
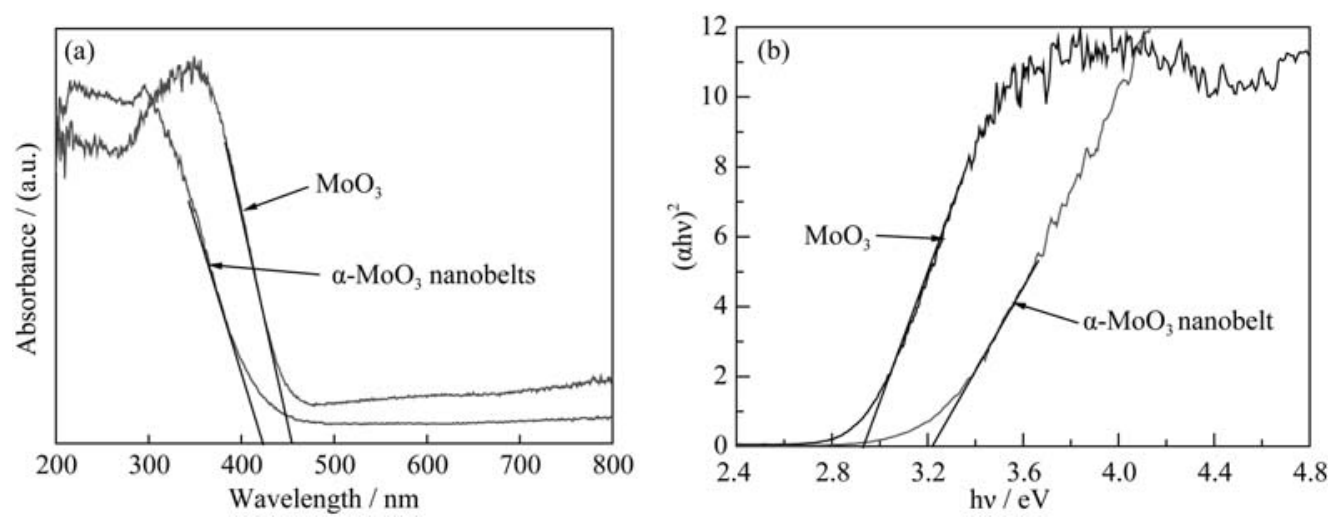

图 3 商品 $\mathrm{MoO}_{3}$ 和 $\alpha-\mathrm{MoO}_{3}$ 的 UV-Vis 漫反射吸收光谱(a) 和( $\left.\alpha \mathrm{hv}\right)^{2}-\mathrm{hv}$ 图(b)

Fig. 3 UV-Vis diffuse reflectance spectra (a) and $(\alpha \mathrm{h} v)^{2} v s \mathrm{~h} v$ plats (b) of commercial $\mathrm{MoO}_{3}$ and as-prepared $\alpha$ - $\mathrm{MoO}_{3}$ nanobelts 


\section{3 样品的光催化氧化脱硫活性}

以噻吩的正辛烷溶液模拟 $\mathrm{FCC}$ 汽油, 以 $1 \mathrm{D}$ $\alpha-\mathrm{MoO}_{3}$ 纳米带为光催化剂, 以空气为氧化剂, 金卤 灯为模拟太阳光进行模拟 FCC 的光催化氧化脱硫 的研究。图 4 为催化用量对模拟 FCC 汽油脱硫率的 影响, 由图可知, 随着催化剂用量的增加, 脱硫率 不断提高, 当催化剂用量达到 $1.5 \mathrm{~g} / \mathrm{L}$ 时脱硫效果最 佳; 继续增加催化剂浓度, 脱硫率几乎保持不变。这 是由于在光催化氧化反应中, 随着催化剂浓度增加, 活性位增多, 光利用率增加, 且适量的催化剂能有 效吸收光量子, 并增加对噻吩的吸附, 导致其向光 反应活性中心扩散, 使可见光催化活性迅速提高, 有利于脱硫。当催化剂用量超过 $1.5 \mathrm{~g} / \mathrm{L}$ 时, 继续增 加催化剂的用量, 中间产物氧的活性物种 $\cdot \mathrm{O}_{2}^{-}$, $\mathrm{O}_{2}^{2-}, \cdot \mathrm{OH}$ (简写 $[\mathrm{O}]$ ) 的浓度很快就能达到一定量, 足以保证反应链的引发, 所以脱硫率基本保持不变 ${ }^{[22]}$ 。 另外, $\alpha-\mathrm{MoO}_{3}$ 纳米带对塞吩有很好的吸附作用, 当 添加量为 $1.5 \mathrm{~g} / \mathrm{L}$ 时, $\alpha-\mathrm{MoO}_{3}$ 纳米带吸附脱硫率约为 $17.5 \%$, 连续光照 $240 \mathrm{~min}$, 经乙腈萃取后, 萃余相 中硫含量降低至 $65.5 \mu \mathrm{g} / \mathrm{L}$, 对应的硫脱除率达 $86.9 \%$ 。在混有 $\mathrm{Ba}(\mathrm{OH})_{2}$ 溶液的试管中, 其水相和 油相的试管壁上产生大量的白色沉淀, 取其少量 加入 $\mathrm{HNO}_{3}$, 沉淀部分溶解, 说明生成的产物中含 有 $\mathrm{SO}_{3}$ 和 $\mathrm{CO}_{2}$ 。

实验对比了自制 $\alpha-\mathrm{MoO}_{3}$ 纳米带、商品 $\mathrm{MoO}_{3}$ 和商品二氧化钛 (P25)的脱硫活性, 如图 5 所示。实 验结果表明, 在相同实验条件下, 商品 $\mathrm{MoO}_{3}$ (分析 纯)和商品二氧化钛(P25)光照 $300 \mathrm{~min}$, 对模拟 FCC 汽油的脱硫率分别为 $61.2 \%$ 和 $69.1 \%$; 而 $\alpha-\mathrm{MoO}_{3}$ 纳 米带光照 $240 \mathrm{~min}$ 的脱硫率可达 $86.9 \%$ 。催化剂的形 貌是影响催化剂活性的重要因素, 形貌的改变影响 尺寸、比表面积和带隙的变化, 从而影响入射光的利

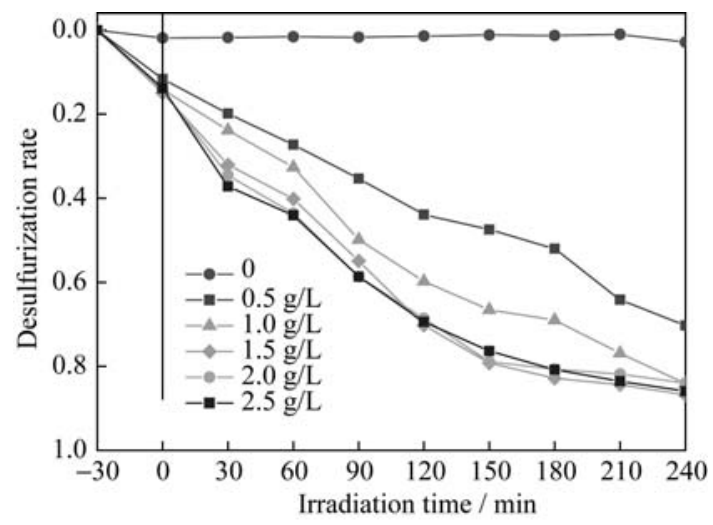

图 4 催化剂用量对 FCC 汽油光催化氧化脱硫活性的影响 Fig. 4 Photocatalytic activity of photocatalys with different concentrations under visible-light irradiation

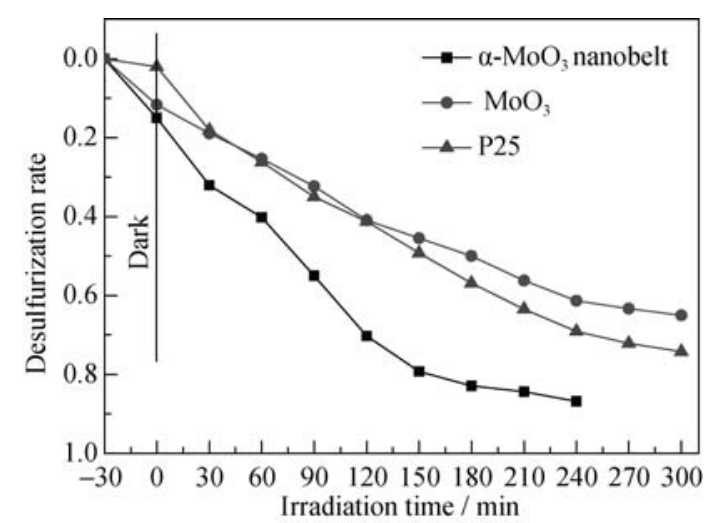

图 5 可见光照射下 $\alpha-\mathrm{MoO}_{3}$ 纳米带与商品 $\mathrm{MoO}_{3}$ 和 $\mathrm{P} 25$ 的光 催化氧化脱硫活性对比

Fig. 5 Comparsion of visible-light-driven photocatalytic activity of $\alpha-\mathrm{MoO}_{3}$ nanobelt, commercial $\mathrm{MoO}_{3}$ and $\mathrm{P} 25$

用率 ${ }^{[23]}$ 。一维纳米带结构有利于光生电子-空穴对 的快速定向传输, 可以延长载流子的寿命, 从而提 高光催化效率 ${ }^{[24-25]}$ 。

\section{4 光催化氧化脱硫机理}

$\alpha-\mathrm{MoO}_{3}$ 纳米带光催化氧化脱硫机理 ${ }^{[26]}$, 如图 6 所示, 光照条件下, $\alpha-\mathrm{MoO}_{3}$ 纳米带吸收 $\geqslant$ 禁带宽度 能量的光时, 价带电子被激发至导带 $(\mathrm{CB})$, 而在价 带(VB)留下空穴。噻吩硫原子上的孤对电子可被纳 米带表面的空穴捕获，形成基态阳离子 $\mathrm{C}_{4} \mathrm{H}_{4} \mathrm{~S} \cdot{ }^{+}$, 而 激发至导带的电子与 $\mathrm{O}_{2}$ 和 $\mathrm{H}_{2} \mathrm{O}$ 作用, 形成具有氧 化性的 $\cdot \mathrm{O}_{2}^{-} 、 \mathrm{O}_{2}^{2-}$ 和 $\cdot \mathrm{OH}$ 等氧的活性物种(简写 $[\mathrm{O}]$ ); 这些活性物种与 $\mathrm{C}_{4} \mathrm{H}_{4} \mathrm{~S}^{+}$结合形成极性的氧化噻吩, 氧化噻吩经过萃取除去。此外, 这些活性 $[\mathrm{O}]$ 还与 $\mathrm{C}_{4} \mathrm{H}_{4} \mathrm{SO}_{2}$ 结合转化为 $\mathrm{CO}_{2} 、 \mathrm{SO}_{3}$ 和 $\mathrm{H}_{2} \mathrm{O}$, 具体反应过 程如下列方程所示:

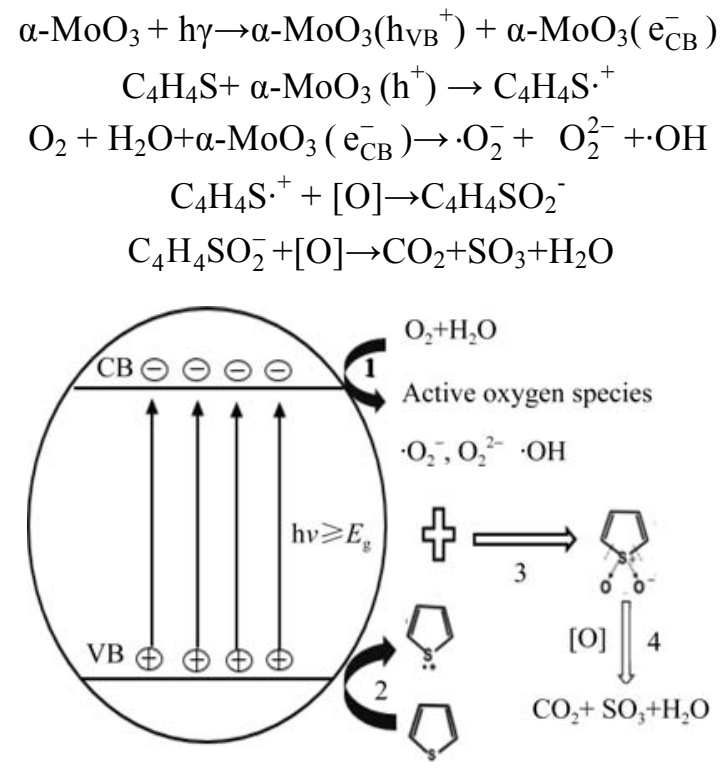

图 $6 \alpha-\mathrm{MoO}_{3}$ 纳米带光催化氧化噻吩机理图

Fig. 6 Schematic diagram of mechanism for the photocatalytic oxidation of thiophene on $\alpha-\mathrm{MoO}_{3}$ nanobelt 


\section{3 结论}

$1 \mathrm{D} \alpha-\mathrm{MoO}_{3}$ 纳米带是可见光响应的光催化剂, 与商品 $\mathrm{MoO}_{3}$ 和二氧化钛( P25)相比, 其在可见光区 光催化氧化脱硫活性更高。当 $1 \mathrm{D} \alpha-\mathrm{MoO}_{3}$ 用量为 $1.5 \mathrm{~g} / \mathrm{L}$, 反应 $240 \mathrm{~min}$ 后脱硫率达到 $86.9 \%$ 。实验结 果为探索 $\mathrm{MoO}_{3}$ 的实际应用提供新的思路。

\section{参考文献:}

[1] GRANGER P, PARVULESCU V I. Catalytic $\mathrm{NO}_{x}$ abatement systems for mobile sources: from three-way to lean burn after-treatment technologies. Chemical Reviews, 2011, 111(5): 3155-3207.

[2] LIU P, RODRIGUEZ J A, ASAKURA T, et al. Desulfurization reactions on $\mathrm{Ni}_{2} \mathrm{P}(001)$ and $\alpha-\mathrm{Mo}_{2} \mathrm{C}(001)$ surfaces: complex role of $\mathrm{P}$ and C sites. Journal of Physical Chemistry. B, 2005, 109: 4575-4583.

[3] ZHANG WEI, XIAO JING, WANG XUN, et al. Oxidative desulfurization using in-situ-generated peroxides in diesel by light irradiation. Energy and Fuels, 2014, 28(8): 5339-5344.

[4] SHIRAISHI Y, TAKI Y, HIRAI T, et al. Visible light-induced desulfurization technique for light oil. Chemical Communications, 1998(23): 2601-2602.

[5] SHIRAISHI Y, HIRAI T, KOMASAWA I. TiO ${ }_{2}$-mediated photocatalytic desulfurization process for light oils using an organic two-phase system. Journal of Chemical Engineering of Japan, 2002, 35(5): 489-492.

[6] NA PING, ZHAO BAO-LIN, GU LIN-YUAN, et al. Deep desulfurization of model gasoline over photoirradiated titanium-pillared montmorillonite. Journal of Physics and Chemistry of Solids, 2009, 70(12): 1465-1470.

[7] SAMOKHVALOV A. Desulfurization of real and model liquid fuels using light: photocatalysis and photochemistry. Catalysis Reviews, 2012, 54(3): 281-343.

[8] LI FA-TANG, LIU YING, SUN ZHI-MIN, et al. Photocatalytic oxidative desulfurization of dibenzothiophene under simulated sunlight irradiation with mixed-phase $\mathrm{Fe}_{2} \mathrm{O}_{3}$ prepared by solution combustion. Catalysis Science and Technology, 2012, 2(7): 1455.

[9] WANG CHAO, ZHU WEN-SHUAI, XU YE-CHAI, et al. Preparation of $\mathrm{TiO}_{2} / \mathrm{g}-\mathrm{C}_{3} \mathrm{~N}_{4}$ composites and their application in photocatalytic oxidative desulfurization. Ceramics International, 2014, 40(8): 11627-11635.

[10] ZHANG WEI, ZHANG HONG, XIAO JING, et al, Carbon nanotube catalysts for oxidative desulfurization of a model diesel fuel using molecular oxygen. Green Chemistry, 2014, 16: 211-220.

[11] CHEN DE-LIANG, LIU MIN-NA, YIN LI, et al. Single-crystalline $\mathrm{MoO}_{3}$ nanoplates: topochemical synthesis and enhanced ethanol-sensing performance. Journal of Materials Chemistry, 2011, 21(25): 9332-9342.

[12] GAO BIN, FAN HUI-QING, ZHANG XIAO-JUN. Hydrothermal synthesis of single crystal $\mathrm{MoO}_{3}$ nanobelts and their electrochemi- cal properties as cathode electrode materials for rechargeable lithium batteries. Journal of Physics and Chemistry of Solids, 2012, 73(3): 423-429.

[13] WANG ZHI-YU, MADHAVI SRINIVASAN, LOU XIONG-WEN. Ultralong $\alpha-\mathrm{MoO}_{3}$ nanobelts: synthesis and effect of binder choice on their lithium storage properties. Journal of Physical Chemistry C, 2012, 116(23): 12508-12513.

[14] SHAKIR I, CHOI J H, SHAHID M, et al. $\mathrm{MoO}_{3}$-MWCNT nanocomposite photocatalyst with control of light-harvesting under visible light and natural sunlight irradiation. Journal of Materials Chemistry, 2012, 22(38): 20549-20553.

[15] JIANG JIAN-BO, LIU JIN-LONG, PENG SAN-JUN, et al. Facile synthesis of $\alpha-\mathrm{MoO}_{3}$ nanobelts and their pseudocapacitive behavior in an aqueous $\mathrm{Li}_{2} \mathrm{SO}_{4}$ solution. Journal of Materials Chemistry A, 2013, 1(7): 2588-2594.

[16] SHAKIR I, SHAHID M, KANG D J. $\mathrm{MoO}_{3}$ and $\mathrm{Cu}_{0 .}{ }_{33} \mathrm{MoO}_{3}$ nanorods for unprecedented UV/visible light photocatalysis. Chemical Communications, 2010, 46(24): 4324-4326.

[17] CHENG LIANG, SHAO MING-WANG, WANG XIU-HUA, et al. Single-crystalline molybdenum trioxide nanoribbons: photocatalytic, photoconductive, and electrochemical properties. Chemistry-A European Journal, 2009, 15(10): 2310-2316.

[18] CHEN YU-PING, LU CHUN-LIANG, XU LIN, et al. Singlecrystalline orthorhombic molybdenum oxide nanobelts: synthesis and photocatalytic properties. Crystal Engineering Communications, 2010, 12(11): 3740-3747.

[19] ZHAO YE, LIU JING-GUO, ZHOU YA, et al. Preparation of $\mathrm{MoO}_{3}$ nanostructures and their optical properties. Journal of Physics: Condensed Matter, 2003, 15(35): L547-L552.

[20] THONGTEM T, PHURUANGRAT A, THONGTEM S. Free surfactant synthesis of microcrystalline CdS by solvothermal reaction. Materials Letters, 2007, 61(14/15): 3235-3238.

[21] SINAIM H, HAM D J, LEE J S, et al. Free-polymer controlling morphology of $\alpha-\mathrm{MoO}_{3}$ nanobelts by a facile hydrothermal synthesis, their electrochemistry for hydrogen evolution reactions and optical properties. Journal of Alloys and Compounds, 2012, 516: 172-178.

[22] ZHAO DI-SHUN, LIU CUI-WEI, MA SI-GUO. Oxdain deulurztion from fluld catalytic cracking gasoline via photocatalysis. Chemical Journal of Chinese Universities, 2006, 27(4): 692-696.

[23] TONG HUA, OUYANG SHUXIN, BI YING-PU, et al. Nanophotocatalytic materials: possibilities and challenges. Advanced Materials, 2012, 24(2): 229-251.

[24] SHEN GUO-ZHEN, CHEN DI. One-dimensional nanostructures for photodetectors. Recent Patents on Nanotechnology, 2010, 4(1): 20-31.

[25] CHATterJeE S, BHATTACHARYYA K, AYYUB P, et al. Photocatalytic properties of one-dimensional nanostructured titanates. The Journal of Physical Chemistry C, 2010, 114(20): 9424-9430.

[26] LIN FENG, LI CAN, WANG DON GE, et al. Photocatalytic oxidation of thiophene on $\mathrm{BiVO}_{4}$ with dual co-catalysts $\mathrm{Pt}$ and $\mathrm{RuO}_{2}$ under visible light irradiation using molecular oxygen as oxidant. Energy and Environmental Science, 2012, 5(4): 6400-6406. 